\title{
Using the head as a mould for cranioplasty with methylmethacrylate
}

\author{
G. M. Bot, N. J. Ismail, B. Usman, D. J. Shilong, J. O. Obande, S. Aliu, D. Sale, B. B. Shehu \\ Department of Neurosurgery, Regional Centre for Neurosurgery, Usmanu Danfodiyo University Teaching Hospital, Sokoto, Nigeria
}

\begin{abstract}
Methacrylate is a valuable tool to the neurosurgeon, even though it is currently being replaced by custom bone. During cranioplasty in the absence of custom bone, which is preformed based on the patients imaging, one has to make a cast to cover the cranial defect with or without the use of a mould. A good artificial skull outline is necessary for prevention of implant extrusion and acceptable cosmetic outcome. Using the patients head as a mould is a simple, cheap, and useful technique. An incision is made, and either a craniectomy or an attempt at skull elevation or separation of the scalp from dura is done based on the indication for the cranioplasty. The methacrylate monomer is mixed with its solvent. It is placed in between a sliced glove and then thinned out. Several layers of drapes are placed on the patients head, the acrylate which is in between the gloves is then placed on the drapes. As soon as it starts setting and the required shape obtained, it is removed and place on the sterile tray. It is then anchored and the wound closed. This technique produces good cosmetic outcome. However, the head must be properly protected from the risk of burns from the exothermic reaction. The technique is described in a 40-year-old driver who had a compound depressed skull fracture. He had a methylmethacrylate cranioplasty in the $9^{\text {th }}$ week post trauma after allowing for wound healing. We recommend that this technique may be used in centers where custom bone is either too expensive or not available during cranioplasty in order to obtain a good outcome.
\end{abstract}

Key words: Acrylate, cranial defects, cranioplast, cranioplasty, decompressive craniectomy, mould, head, polymethylmethacrylate

\section{Introduction}

Methacrylate is a methylester of methacrylic acid (monomer). Methacrylate also known as methyl methacrylate was produced in commercial quantities in 1927 and it has several chemical forms. It consists of the liquid or monomer and the powder or polymer, when these substances are combined the form an acrylic resin in a process known as polymerization. ${ }^{[1]}$

Methyl methacrylate monomer is a colorless liquid which is volatile and has an acrid fruity smell, with a boiling temperature of $212^{\circ} \mathrm{F}\left(100^{\circ} \mathrm{C}\right)$. It has the

\begin{tabular}{|l|l|}
\hline \multicolumn{2}{|c|}{ Access this article online } \\
\hline Quick Response Code: & Website: \\
\hline & www.ruralneuropractice.com \\
\cline { 2 - 2 } & \\
\hline & \\
\hline
\end{tabular}

empirical formula $\mathrm{C}_{5} \mathrm{H}_{8} \mathrm{O}_{2} \cdot{ }^{\left[{ }^{[2]}\right.}$ Inhibitors are usually added for storage and transport, such as methyl ether of hydroquinone and hydroquinone, although phenolic inhibitors such as dimethyl tert-butylphenol could be used. The monomer also contains an activator called dimethyl-para-otoludine (DMpT), although as at 2005 only one commercial bone cement had a different activator, dimethylamino phenyl ethanol (DMAPE). ${ }^{[3]}$

The bone cement powder consists of a spherical polymer powder made of polymethylmethacrylate (PMMA) or methyl methacrylate copolymer containing the initiator dibenzoyl peroxide (BPO), a radiopacifier such as Zirconium dioxide or barium sulfate and others substances that are optional including antibiotics and dyes. The radiopacifier, the antibiotics, and the dye do not take part in the curing process which is called "radical polymerization." The curing of bone cement consists mainly of radical polymerization, heat formation, and volume shrinkage. Two important processes from a scientific viewpoint occur; initially, the polymer powder takes up the monomer liquid in a physical process

Address for correspondence:

Dr. Gyang M. Bot, Regional Centre for Neurosurgery, Usmanu Danfodiyo University Teaching Hospital, P.M.B. 2370, Sokoto, Nigeria.

E-mail: gyangbot@yahoo.co.uk 
of swelling and dissolution. Thereafter, an initiation reaction occurs involving the initiator from the polymer powder $\mathrm{BPO}$ and an activator from the liquid monomer $\mathrm{DMpT}$, which lead to the production of free radicals begins the polymerization process by adding to the polymerizable double bond of the monomer. The polymerization process is an exothermic reaction. Four phases have been described in the handling of bone cement, which are the mixing (up to $1 \mathrm{~min}$ ), waiting (several minutes), working (2-4 min), and hardening phases (1-2 $\mathrm{min}) .^{[3]}$

This hard structure can be used as an artificial bone during cranioplasty. Methacrylate was first use by Zander in 1940. ${ }^{[1]}$ Acrylic resins were used extensively during the Second World War for cranioplasty. Kahn and Kerr in 1943 used preformed acrylic plates for skull defects. ${ }^{[4]}$ In 1945, Small and Graham performed cranioplasty in two stages; first a direct impression of the skull defect was taken, laboratory work done, and then, a plate was inserted at the second stage of operation. ${ }^{[4]}$ In 1948, Oliver and Blaine used it on three cases as a single-stage cranioplasty; however, Woringer became the main advocate in 1951 for single-stage crainioplasty after presenting 15 cases. ${ }^{[5,6]}$

Several materials can be used for cranioplasty and they can be classified into natural and synthetic. The natural materials can be further classified based on the source into autografts, allograft, and xenograft. While the synthetic materials (alloplast) can be classified into metallic and nonmetallic materials. The autografts can be obtained from the cranium, ribs, scapula, tibia, ilium, and fascia, of the same person, while the allografts are from genetically different organisms (persons) of the same species and xenografts are from animal species (different species). The nonmetallic materials include methylmethacrylate, hydroxyapatite, polyethylene and silicone, chorale, ceramic, and Cortoss. The metallic materials include titanium, stainless steel, gold, aluminum, tantalum, lead, platinum, vitallium, and ticonium. It is important to state that some of these materials are not in current use. The commonest materials currently used to for cranioplasty include methylmethacrylate, titanium mesh and plates, hydroxyapetite, and the cranium (either stored or as a split calvarial bone graft). ${ }^{[7,8]}$

Currently, hydroxy appetite, also known as custom bone, is in use. It is preformed for a specific patient based on CT or MRI reconstruction of the defective part of the skull. It also has the advantage of having pores within it, which allow for osteoblast to be laid over the custom bone. However, the custom bone is expensive and not readily available in low-income countries. Hence, the need to improve on the outcome of what is available is necessary. Patient with depressed skull fracture, fibrous dysplasia or those who have had decompressive craniectomy and the bone could not be stored may need cranioplasty, either from an autogenous or synthetic source. A good mould is necessary to produce good cosmetic outcome as well as reduce the risk of extrusion of the implant, which is usually as a result of pressure from the sharp edge of the acrylate if not molded in such a way that it lies flush with the bone and overlying skin.

\section{Materials and Methods}

In a patient who has met the indication for a cranioplasty under general anesthesia, a bicoronal incision or suitable incision is used to expose the area required for crainioplasty, such that the suture line does not lie on the implant. A craniectomy is done in the case of a fibrous dysplasia or an attempt at elevation is made if necessary or the scalp is separated from dura in the case of patients with previous decompressive craniectomy. The methacrylate is prepared by mixing the methyl methacrylate monomer (the liquid form) with polymethylmethacrylate (PMMA) or methyl methacrylate copolymer (which is in powder form) to obtain the appropriate consistency, which is then placed between a sliced glove and thinned out. After several layers of dapes (4-5) are placed on the patient's head, the acrylate is then wrapped in a towel and placed over the patient's head, as shown in Figures 1 and 2. This is to avoid burns from the exothermic reaction that occurs when the monomer is converted to a polymer during the process of polymerization. As soon as it starts setting and the required shape is obtained, it is removed and place on the sterile tray. The methacrylate is then perforated with a towel clip or drill with the adjacent bone following which the acrylate is anchored with either silk 1 or nylon 1 suture. The scalp is returned and sutured in two layers, initially the galea and subsequently the skin with vicryl and nylon sutures, respectively, by continuous stitch.

\section{Result}

A 40-year-old driver admitted with a wound on the forehead, following road traffic accident sustained 5 days prior to presentation. He was an unrestrained driver of a 10-seater bus that had a head on collusion with a saloon car at high speed. The patient was thrown out of the vehicle and lost consciousness which he regained within $24 \mathrm{~h}$. He bled from a laceration on the forehead and had a deformity in the forehead with partial loss of the nose. No history suggestive of injury to other organs or systems. He was initially resuscitated in another hospital before being referred to our hospital. Examination revealed a deep laceration at the mid frontal region, which was $4 \mathrm{~cm}$ deep 


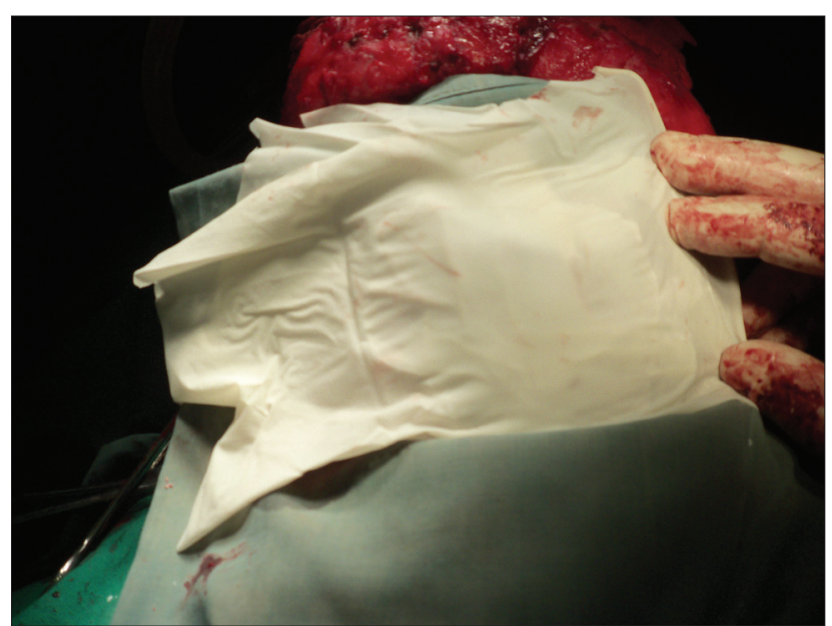

Figure 1: Acrylate placed between the sliced gloves and thinned out with it placed over layers of drapes

and $6 \mathrm{~cm}$ long which was infected with discharge. He had right-eye ecchymosis, left-eye enophthalmos with loss of vision on the left eye. His GCS was 15/15 with no other neurological deficits. A diagnosis of mild head injury with compound depressed frontal skull fracture was made and his admitting packed cell volume was $19 \%$ for which he had 3 units of blood transfused. He was co-managed with ENT, plastic surgeon, and ophthalmology. He had wound dressing initially until the wound healed. Afterwards he was worked up for a cranioplasty with methyl methacrylate using the head as a mould technique; this was done on the $9^{\text {th }}$ week of admission. He did well and was discharged on the $12^{\text {th }}$ day postoperatively. He came for follow up 4 months after discharge with flap necrosis but he decline admission for financial reasons.

The index patient was the first amongst our patients that was managed with this technique, though he had a flap necrosis it was possibility due to the poor quality of overlying skin.

The cosmetic outcome is good with a relatively reduced risk of the implant extruding as a result of a fairly good contour obtained from the mould (the patient's head). However, it must be strongly emphasized that care must be taken to avoid burns.

\section{Discussion}

The presence of a cranial defect produces not only an aesthetic abnormality, but also a functional problem. The functional abnormality is known as the "syndrome of the trephined" and the symptoms include headache, dizziness, irritability, depression, discomfort at defect edge, contralateral hemiparesis, intolerance to noise or vibration, and anxiety. Hence, the purpose of

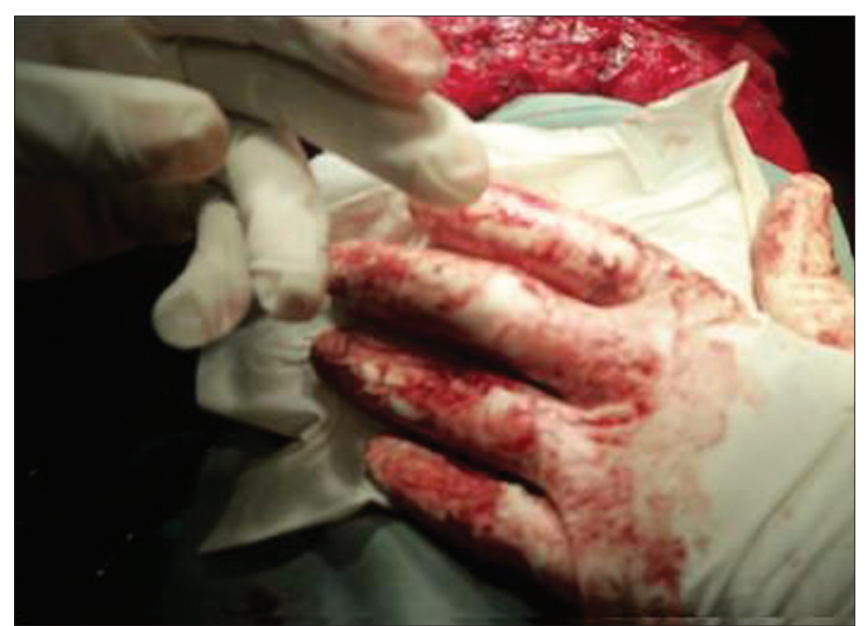

Figure 2: Using the head as a mould

cranioplasty is both for cosmetics as well as improving the patient's neurology. ${ }^{[9,10]}$

Cranioplasty could be achieved by the use of metals, grafts, and plastic based materials such as acrylate. The characteristics of a good synthetic material include, biocompatibility, low or non thermal conductivity, similar weight or lighter than bone, strong enough to withstand functional stress, easy to use, cheap, available, and should not produce an artifact on computed tomography (CT) scan or Magnetic resonance imaging (MRI). ${ }^{[1]}$ Polymethylmethacrylate is the most commonly used cranioplast; however, polyethylmethacrylate has the advantages of easy handling, lower polymerization temperature, and greater elasticity. A combination of polyethylmethacrylate and hydroxyapatite has the additional benefit of a physical resistance similar to bone and the ability of collagen bridges to form between the bone and hydroxyapatite. ${ }^{[11]}$

The mould could be made from either on the patients head with an intact skin which is shaved and draped, from the skull or the previous craniectomy site if it is not sunken. In the presence of a good exposure and if the defect is not so large, it can be placed on the patients skull which is relatively more resilient after applying the drapes. In cases of large cranial defects (i.e., greater than $10 \mathrm{~cm}$ by $10 \mathrm{~cm}$ or frontotemporoparietal craniotomies), the previous craniectomy site could be used if it is not sunken, but in large defects with sunken craniectomy sites, the cranial vault and adjourning contralateral side may be used.

Based on the four stages of handing of methyl methacrylate stated earlier, we recommend that as soon as the hardening stage commences and the mould has been formed it can be removed from the operation site. Even though the maximum temperature reached was not recorded in this case. We recommend that the mould should be placed on 
the patient head for about 3-6 min, after the working phase but the most important determinant is the temperature. However, the added advantage of the multiple layer draping is that it prevents the underlying tissue from being injured even if it is left for the period of hardening to occur.

In the absence of special silicone rubber mould or the use of plaster of paris and the wax elimination technique, as described by Rotaru et al. and Abdulai et al., respectively ${ }^{[1,12]}$ the head of the patient can be used as a mould after adequate padding. This method provides a simple and fast technique for cranioplasty.

We therefore recommend that in centers where custom bone is not available the patient's skull is a handy tool in the production of suitable cranioplast with good cosmetic outcome and with a good outline that is likely to reduce the risk of implant protrusions with subsequent implant exposure.

\section{References}

1. Sanan A, Haines SJ. Repairing holes in the head: A history of cranioplasty. Neurosurgery 1997;40:588-603. International Programme on Chemical Safety-World Health Organization; 1998.

2. Concise International Chemical Assessment Document No. 4-Methyl
Methacrylate. Available from: http://www.inchem.org/documents/ cicads/cicads/cicad04.htm [Last cited on 2013 Jan 15].

3. Kühn KD, Ege W, Gopp U. Acrylic Bone Cement: Composition and Properties. Ortho Clin North Am 2005;36:18-28.

4. Robinson RG, Macalister AD. Acrylic cranioplasty a simple one-stage method using a cold curing material. Br J Surg 1954;42:312-5.

5. Oliver LC, Blaine G. A new one-stage method of cranioplasty with acrylic plastic. Med Press 1948;220:167-8.

6. Woringer E, Schweig B, Brogly G, Schneider J. New ultramodern technique of repair of cranial damages with acrylic resins; advantage of acrylic resins over tantalum. Rev Neurol 1951;85:527-35.

7. Agrawal A, Garg LN. Split calvarial bone graft for the reconstruction of skull defects. J Surg Tech Case Report 2011;3:13-6.

8. Aydin S, Kucukyuruk B, Abuzayed B, Aydin S, Sanus GZ. Cranioplasty: Review of materials and techniques. J Neurosci Rural Pract 2011;2:162-7.

9. Dujovny M, Aviles A, Agner C, Fernandez P, Charbel FT. Cranioplasty: Cosmetic or therapeutic? Surg Neurol 1997;47:238-41.

10. Dujovny M, Agner C, Aviles A: Syndrome of the trephined: Theory and facts. Crit Rev Neurosurg 1999;24:271-8.

11. Rotaru H, Baciut M, Stan H, Bran S, Chezan H, Iosif A, et al. Silicone rubber mould cast polymethylacrylate-hydroxyapatite plate used for repairing skull defect. J Craniomaxillofac Surg 2006;34:242-6.

12. Abdulai AE, Iddrissu MI, Dakurah TK. Cranioplasty using polymethyl methacrylate implant constructed from an alginate impression and wax elimination technique. Ghana Med J 2006;40:16-21.

How to cite this article: Bot GM, Ismail NJ, Usman B, Shilong DJ, Obande JO, Aliu S, Sale D, Shehu BB. Using the head as a mould for cranioplasty with methylmethacrylate. J Neurosci Rural Pract 2013;4:471-4.

Source of Support: Nil. Conflict of Interest: None declared. 